\title{
Rapid and Efficient Removal of Perfluorooctanoic Acid from Water with Fluorine-Rich Calixarene-Based Porous Polymers
}

\author{
Dinesh Shetty, ${ }^{\mathrm{a}, \mathrm{b}, \mathrm{c}, \mathrm{f}}$ Ilma Jahović, ${ }^{\mathrm{a}, \#, \dagger}$ Tina Skorjanc, ${ }^{\mathrm{a}}$ Turan Selman Erkal, ${ }^{\mathrm{d}}$ Liaqat Ali, ${ }^{\mathrm{e}}$ Jesus Raya, \\ Zouhair Asfari, ${ }^{\mathrm{g}}$ Mark A. Olson, ${ }^{\text {h,i }}$ Serdal Kirmizialtin, ${ }^{\mathrm{a}}$ A. Ozgur Yazaydin, ${ }^{\mathrm{d}}$ and Ali Trabolsi ${ }^{{ }^{*}}$ \\ a Science Division, New York University Abu Dhabi, Saadiyat Island, Abu Dhabi, UAE \\ ${ }^{\mathrm{b}}$ Department of Chemistry, Khalifa University of Science and Technology, P.O. Box: 127788, Abu Dhabi, UAE \\ ${ }^{\mathrm{c}}$ Center for Catalysis and Separations, Khalifa University of Science and Technology, P.O. Box 127788, Abu Dhabi, UAE \\ ${ }^{d}$ Department of Chemical Engineering, University College London, Torrington Place, London WC1E 7JE, UK \\ ${ }^{\text {e }}$ Core Technology Platform, New York University Abu Dhabi, Saadiyat Island, Abu Dhabi, UAE \\ ${ }^{\mathrm{f}}$ Membrane Biophysics and NMR, Institute of Chemistry, UMR 7177, University of Strasbourg, CNRS, Strasbourg, France \\ g Laboratoire de Chimie Analytique et Sciences Séparatives, Institut Pluridisciplinaire Hubert Curien, 67087 Strasbourg \\ Cedex, France. \\ ${ }^{\text {h }}$ Department of Chemistry, Northwestern University, 2145 Sheridan Road, Evanston, IL 60208 USA \\ ${ }^{i}$ Institute for Molecular Design and Synthesis, School of Pharmaceutical Science and Technology, Tianjin University, Tian- \\ jin, PR.
}

KEYWORDS: calixarene, porous polymers, perfluorooctanoic acid, Sonogashira-Hagihara coupling, water purification

\begin{abstract}
On account of its non-biodegradable nature and persistence in the environment, perfluorooctanoic acid (PFOA) accumulates in water resources and poses serious environmental issues in many parts of the world. Here, we present the development of two fluorine-rich calix[4]arene-based porous polymers, FCX4-P and FCX4-BP, and demonstrate their utility for the efficient removal of PFOA from water. These materials featured BET surface areas of up to $450 \mathrm{~m}^{2} \mathrm{~g}^{-1}$, which is slightly lower than their non-fluorinated counterparts (up to $596 \mathrm{~m}^{2} \mathrm{~g}^{-1}$ ). FCX4-P removes PFOA at environmentally relevant concentrations with a high rate constant of 3.80 $\mathrm{g} \mathrm{mg}^{-1} \mathrm{~h}^{-1}$ and reaches an exceptional maximum PFOA uptake capacity of $188.7 \mathrm{mg} \mathrm{g}^{-1}$. In addition, it can be regenerated by simple methanol wash and reused without a significant decrease in performance.
\end{abstract}

\section{INTRODUCTION}

Per- and polyfluoroalkyl substances (PFAS) are a class of chemicals that are extensively used in clothing, furniture, cookware, and firefighting industries for their oil, grease, and water repellency. ${ }^{1}$ PFAS contain C-F bonds, which are known to be among the strongest chemical bonds resistant to degradation. ${ }^{2}$ On account of the fact that PFAS-producing companies are facing lawsuits to compensate for the environmental problems caused by these persistent perfluorinated compounds, efficient methods for the removal of PFAS from water are now in high demand. Perfluorooctanoic acid (PFOA) is one of the most commonly utilized PFAS. PFOA concentrations three to four orders of magnitude higher than the lifetime health advisory of $70 \mathrm{ng} \mathrm{L}^{-1}$ determined by the Environmental Protection Agency were discovered in ground and surface waters close to civilian airports and fire-fighting training areas. ${ }^{3}$ Additionally, PFOA was detected in the majority of serum samples collected from populations worldwide, and the most possible exposure pathway was reported to be drinking water. ${ }^{4}$ Therefore, there is a pressing need to find a straightforward, economical, and efficient method to solve the problem of global PFOA and PFAS contamination.
Various oxidative, reductive, photochemical, and electrochemical methods for PFOA sequestration or degradation have been reported. ${ }^{5-8}$ However, PFOA does not lend itself to facile removal techniques; the high stability of $\mathrm{C}-\mathrm{F}$ bonds with the bond dissociation energy of up to $130 \mathrm{kcal} \mathrm{mol}^{-1}$ results in PFOA's resistance to metabolic degradation and conventional decontamination methods. ${ }^{9}, 10$ Certain removal approaches are limited by the sensitivity of the analytical methods used (up to $\mathrm{mg} \mathrm{L}^{-1}$ ) for detection, which is a significant drawback as trace amounts (ng L ${ }^{-1}$ to low $\mu \mathrm{g} \mathrm{L}^{-1}$ ) of PFOA in the environment can induce toxic effects in living organisms. ${ }^{11}$ Many degradation techniques result in the formation of short-chain polyfluorinated alkyl substances of unknown toxicity. ${ }^{12}$ For this reason, significant research has been dedicated to using adsorption for the removal of PFOA from water because adsorbents remove PFOA instead of degrading it. In addition, adsorbents have reasonable rates of removal, low costs, high surface-area-to-volume ratios, and an abundance of adsorption sites. ${ }^{13}$ The most commonly used adsorbent for PFOA removal is activated carbon (AC). Despite being affordable and relatively efficient at removing PFOAs, ACs suffer from low affinity for PFOA at environmentally relevant concentrations,${ }^{14}$ and their regeneration is energy-intensive. ${ }^{15}$ Thus, developing materials with high 
PFOA affinity and ease of regeneration is key to improving the performance of adsorbents used for PFOA removal from water. ${ }^{16}$

One class of materials that can address this challenge is macrocycle-containing porous organic polymers, whose utility can be tailored by the rational choice of organic monomers, which allows for optimization of molecular architecture and hostguest interactions. ${ }^{17,18}$ Recently, $ß$-cyclodextrin-containing polymers showed some affinity for PFOA $\left(Q_{\max }=34 \mathrm{mg} \mathrm{g}^{-1}\right)$ with uptake kinetics comparable to that of commercial $\mathrm{AC}\left(\mathrm{k}_{\mathrm{obs}}=\right.$ $\left.2.88 \mathrm{~g} \mathrm{mg}^{-1} \mathrm{~h}^{-1}\right){ }^{19,20}$ Highly fluorinated covalent organic polymers (COPs) were found to reduce PFOA concentration from 1 $\mu \mathrm{g} \mathrm{L}^{-1}$ to $<10 \mathrm{ng} \mathrm{L^{-1 }}{ }^{19}$ PFOA removal by solid phase extraction $^{21}$ and in packed beds ${ }^{22}$ has also been explored. However, the cyclodextrin polymers have a relatively low $\mathrm{Q}_{\max }$ value and their rate of adsorption is also fairly slow. Therefore, investigation of alternative building blocks for covalent polymers is of high interest.

Herein, we report our efforts to leverage the benefits of porous organic polymers and to introduce porous calixarenebased polymers with fluorinated alkyne-rich linkers that can efficiently remove PFOA from water. ${ }^{23-25}$ Calixarene macrocycles can be exploited for their amphiphilic nature around the rim, ${ }^{26}$ whereas the fluorinated linkers make the overall network even more hydrophobic and enhance $\mathrm{C}-\mathrm{F} \cdots \mathrm{F}-\mathrm{C}$ interactions between the polymers and guest molecules. Furthermore, calixarenes possess a hydrophobic cavity, which can facilitate favorable host-guest interactions. ${ }^{27}$ The current study aims to investigate the synergy between the inherent host-guest chemistry and hydrophobic properties of polycalixarenes with the enhanced hydrophobicity and C-F interactions of fluorinated linkers.

\section{RESULTS}

Given that calixarene-based porous polymers are efficient agents for the removal of various organic micropollutants from water, ${ }^{28}$ we anticipated that introducing fluorine-rich moieties, in the form of perfluoroarene linkers, would enhance the hydrophobic interactions between the adsorbent and PFOA. Such a system would also simultaneously maintain hydrogen bonding between the hydroxyl groups of the macrocycle and PFOA, ${ }^{29}$ thus facilitating PFOA removal from water. In line with our hypothesis, one of the designed polymers, FCX4-P, displayed excellent adsorption ability, which was quantified by HPLCMS/MS/MS. The performance analysis of this material indicated that FCX4-P was a superior adsorbent as compared to commercial $\mathrm{AC}$ and a non-fluorinated analogue of the polymer, CX4-P. Moreover, we found that FCX4-P has one of the fastest reported PFOA adsorption kinetics at environmentally relevant concentrations and one of the highest maximum adsorption capacities for any class of material.

Non-fluorinated polymers used as benchmark materials in this study were synthesized through Sonogashira-Hagihara coupling between tetrabromo-calix[4]arene-tetrol, $\mathbf{1}$, and the nonfluorinated versions of linkers 2 and 3, either 1,4-diethynylbenzene or 4,4'-diethynyl-1,1'-biphenyl, to form CX4-P and CX4BP, respectively. ${ }^{30,31}$ To obtain the fluorinated polymers, the fluorinated versions of linkers $\mathbf{2}$ and $\mathbf{3}$ were first synthesized through Sonogashira-Hagihara coupling reactions between either 1,4-diiodo-2,3,5,6-tetrafluorobenzene or 4-4'-dibromooctafluoro biphenyl with (trimethylsilyl)acetylene (compounds $\mathbf{2} \mathbf{a}$ and $\mathbf{3} \mathbf{b}$ in the SI), followed by deprotection of the silyl groups with tetra-n-butylammonium fluoride to afford the linkers in good yields. ${ }^{32}$ Subsequent Sonogashira-Hagihara coupling of the fluorinated linkers, 2 and 3, with tetrabromo-calix[4] arene-tetrol, $\mathbf{1}$, in a 1:1 mixture of toluene and triethylamine led to the isolation of brown powders, FCX4-P and FCX4-BP, respectively, after heating at $110^{\circ} \mathrm{C}$ for $70 \mathrm{~h}$ (Scheme 1). The resulting polymers were washed with toluene, ethanol, water, tetrahydrofuran, and dichloromethane, and dried under vacuum for 16 hours. All polymers were insoluble in all tested solvents, indicating the formation of cross-linked polymeric networks. Fourier transform infrared (FT-IR) spectra of FCX4-P and FCX4-BP exhibited absorption bands at 1267 and $1257 \mathrm{~cm}^{-1}$, respectively (Figure 1a), which corresponded to $-\mathrm{C}-$ $\mathrm{F}$ stretches. For all four polymers, a broad band corresponding to the $-\mathrm{O}-\mathrm{H}$ stretching vibration of calixarene was observed near $3100 \mathrm{~cm}^{-1}$ (Figure S1 and 1a). The presence of the asymmetric $-\mathrm{C} \equiv \mathrm{C}$ - stretching vibrations near $2250 \mathrm{~cm}^{-1}$ and the absence of the terminal alkyne $-\mathrm{C}-\mathrm{H}$ stretching vibrations at 3300 $\mathrm{cm}^{-1}$ in the polymers confirmed successful crosslinking of the linkers with the brominated calixarene monomers. Further molecular-level characterization of the polymers was achieved by solid state NMR spectroscopy. Solid-state cross-polarization magic angle spinning (CP/MAS) ${ }^{13} \mathrm{C}$ NMR spectra (Figure $1 \mathrm{~b}$ ) exhibited resonances in the range of $105-145 \mathrm{ppm}$, which correspond to the aromatic carbons of the calixarene macrocycle and the fluorinated linkers.

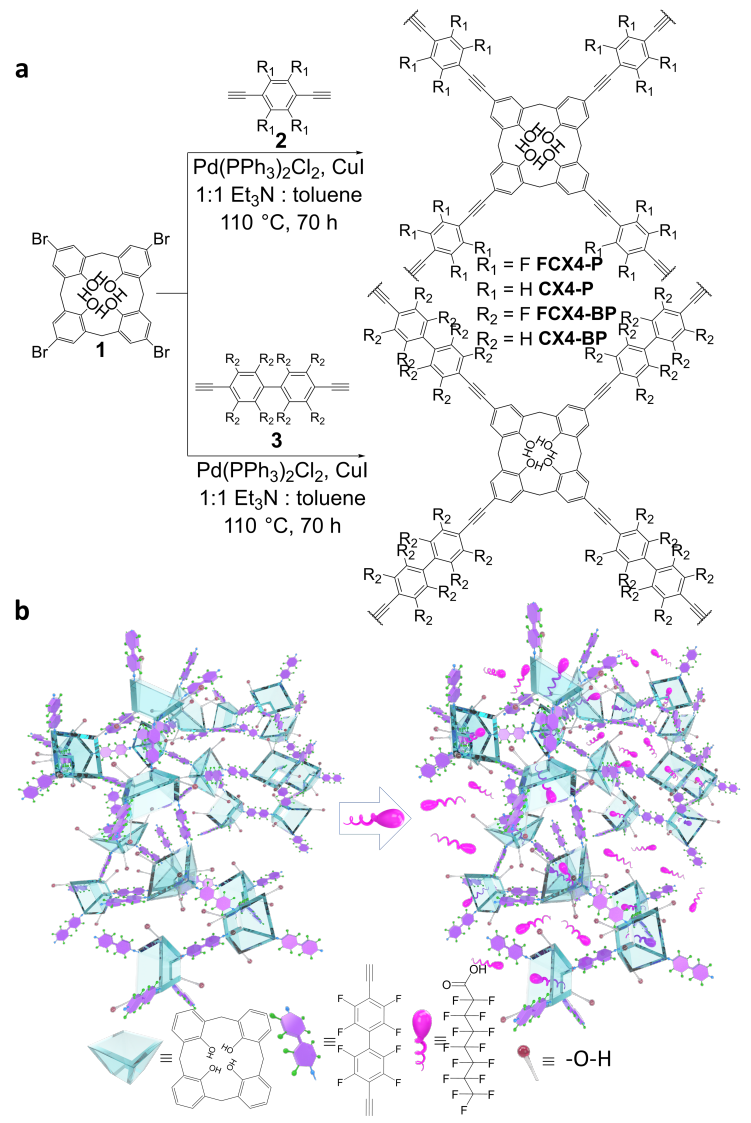

Scheme 1. a) Design strategy for the synthesis of fluorinated and non-fluorinated calix[4] arene polymers, FCX4-P, FCX4-BP, CX4$\mathbf{P}$, and CX4-BP. b) Schematic presentation of representative fluorinated polymer and its utility as PFOA sorbent. Cyan cones correspond to calixarenes, purple hexagons with green endings to the fluorinated linker, pink spirals to PFOA and brown spheres to the $\mathrm{OH}$ groups of calixarene. 
a

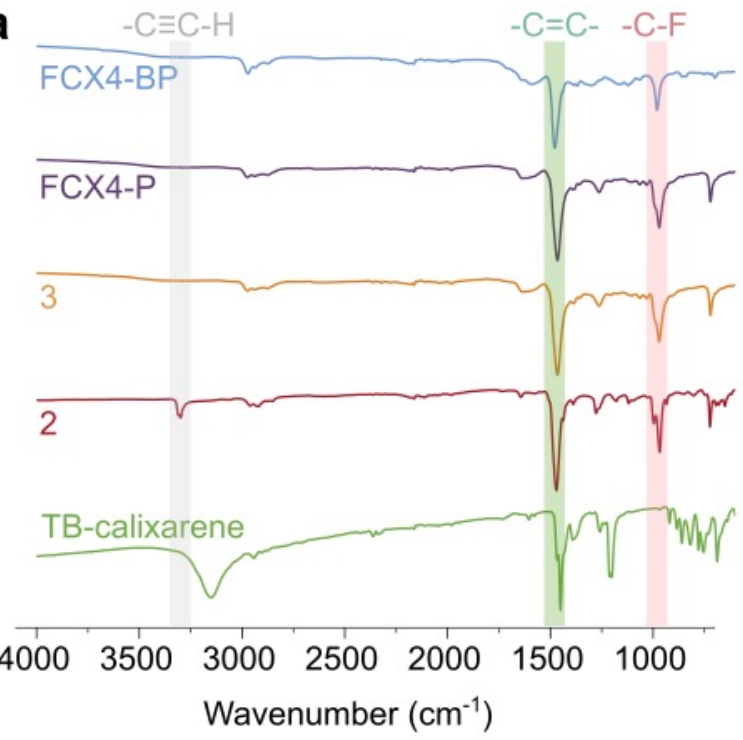

C

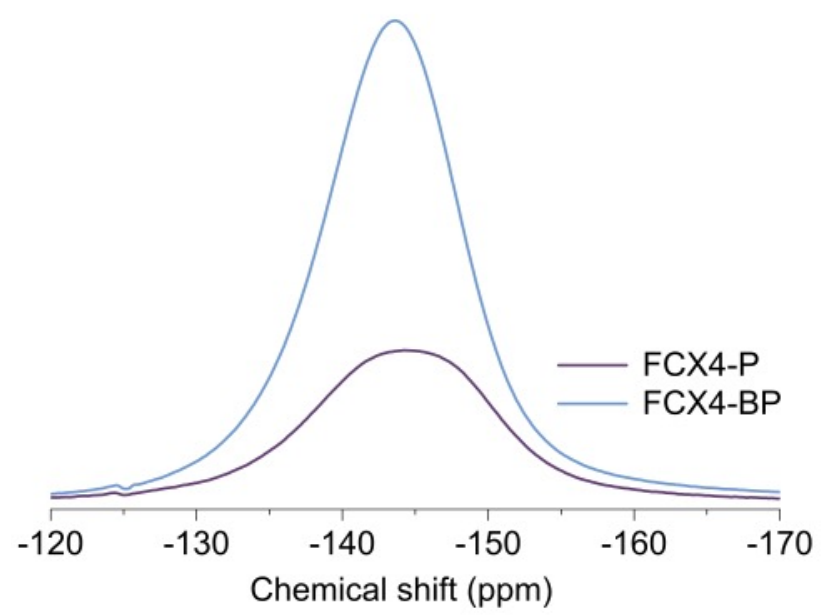

b

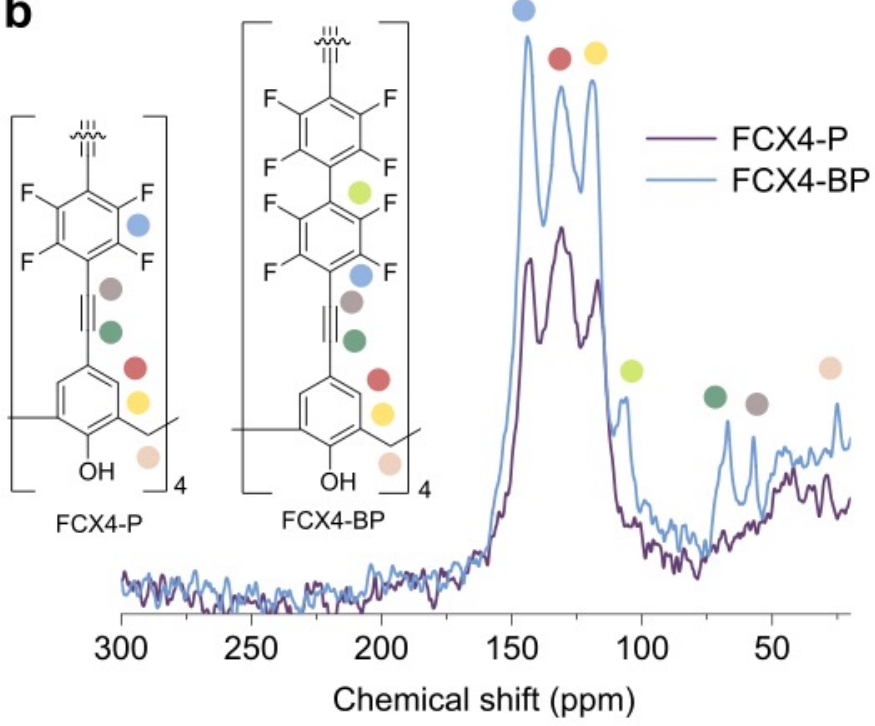

d

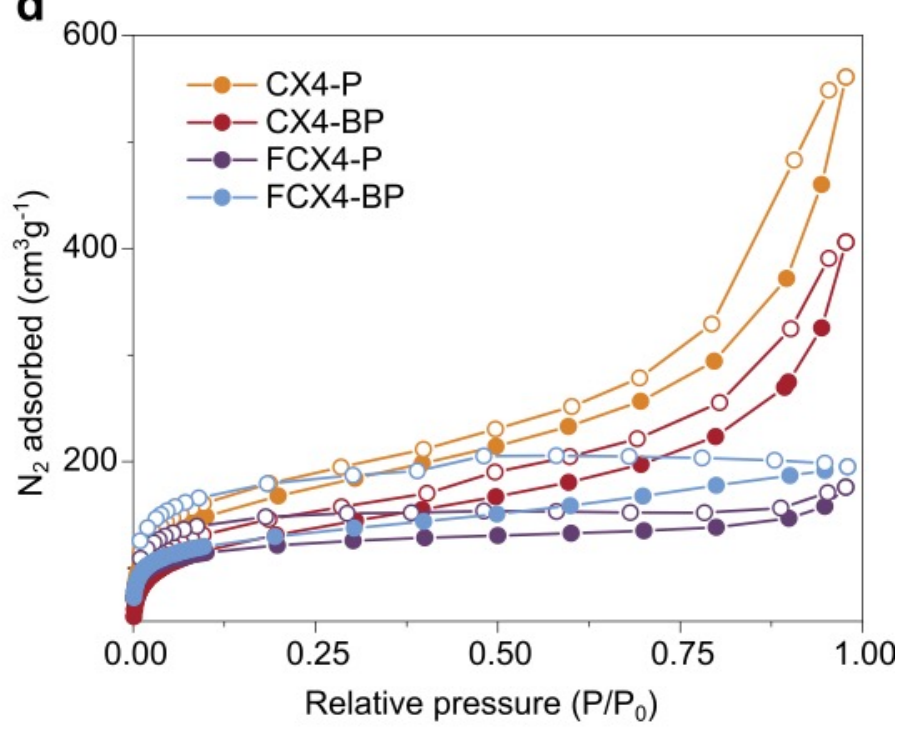

Figure 1. (a) FT-IR spectra of as-synthesized FCX4-P (purple) and FCX4-BP (blue), linkers 2 and 3 (red and orange) and 5,11,17,23-tetrabromo-25,26,27,28-tetrahydroxycalix[4] arene (1) (green); (b) ${ }^{13} \mathrm{C} \mathrm{CP} / \mathrm{MAS}$ NMR spectrum of FCX4-P and FCX4-BP with corresponding peak assignments; (c) ${ }^{19} \mathrm{~F}$ solid-state NMR spectra of FCX4-P and FCX4-BP; (d) $\mathrm{N}_{2}$ adsorption and desorption isotherms for FCX4-P, FCX4-BP and the corresponding non-fluorinated polymers, CX4-P and CX4-BP. ${ }^{31}$

The peak near 25 ppm was assigned to the phenylene carbons $\left(-\mathrm{CH}_{2}-\right)$ of the macrocycle, while the peak near $90 \mathrm{ppm}$ indicated the presence of the acetylene $(-\mathrm{C} \equiv \mathrm{C}-)$ moieties. Solidstate ${ }^{19} \mathrm{~F}$ NMR spectra for both polymers exhibited a signal at $141.4 \mathrm{ppm}$, reflecting the fact that there is only one fluorine environment in both polymers, a characteristic which is due to strong dipolar coupling between neighboring fluorine atoms (Figure 1c).

The morphology of the resulting polymers was analyzed by electron microscopy techniques. Both scanning electron microscopy (SEM) and transmission electron microscopy (TEM) analyses of the polymers revealed the material to be comprised of amorphous clumps (Figure S3). Thermogravimetric analyses (TGA) revealed exceptional thermal stability of the polymers up to $500{ }^{\circ} \mathrm{C}$ (Figure S4). At that temperature, $~ 80 \%$ of fluorinated and $\sim 90 \%$ of non-fluorinated polymers are degraded.
The porosity of the polymers was studied by $\mathrm{N}_{2}$ gas adsorption/desorption measurements following degassing of the samples at $85{ }^{\circ} \mathrm{C}$ for 24 hours (Figure 1d). Fitting of the $\mathrm{N}_{2}$ isotherms to the BET model provided calculated surface areas of 596, 468, 417, and $451 \mathrm{~m}^{2} \mathrm{~g}^{-1}$, for CX4-P, CX4-BP, FCX4-P, and FCX4-BP, respectively. The slightly lower surface area of fluorinated polymers could be explained by the larger size of fluorinated linkers whereby the fluorine atoms occupy more space. Average pore diameters for the materials ranged from 48 to $95 \AA$ (Figure S5), with cumulative pore volumes ranging from 0.11 to $0.73 \mathrm{~cm}^{3} \mathrm{~g}^{-1}$.

To evaluate the PFOA removal efficiency of each polymer, batch adsorption studies were conducted with $1 \mu \mathrm{g} \mathrm{L}^{-1}$ of PFOA, which is comparable to the amount of PFOA present in contaminated water resources. ${ }^{33}$ 

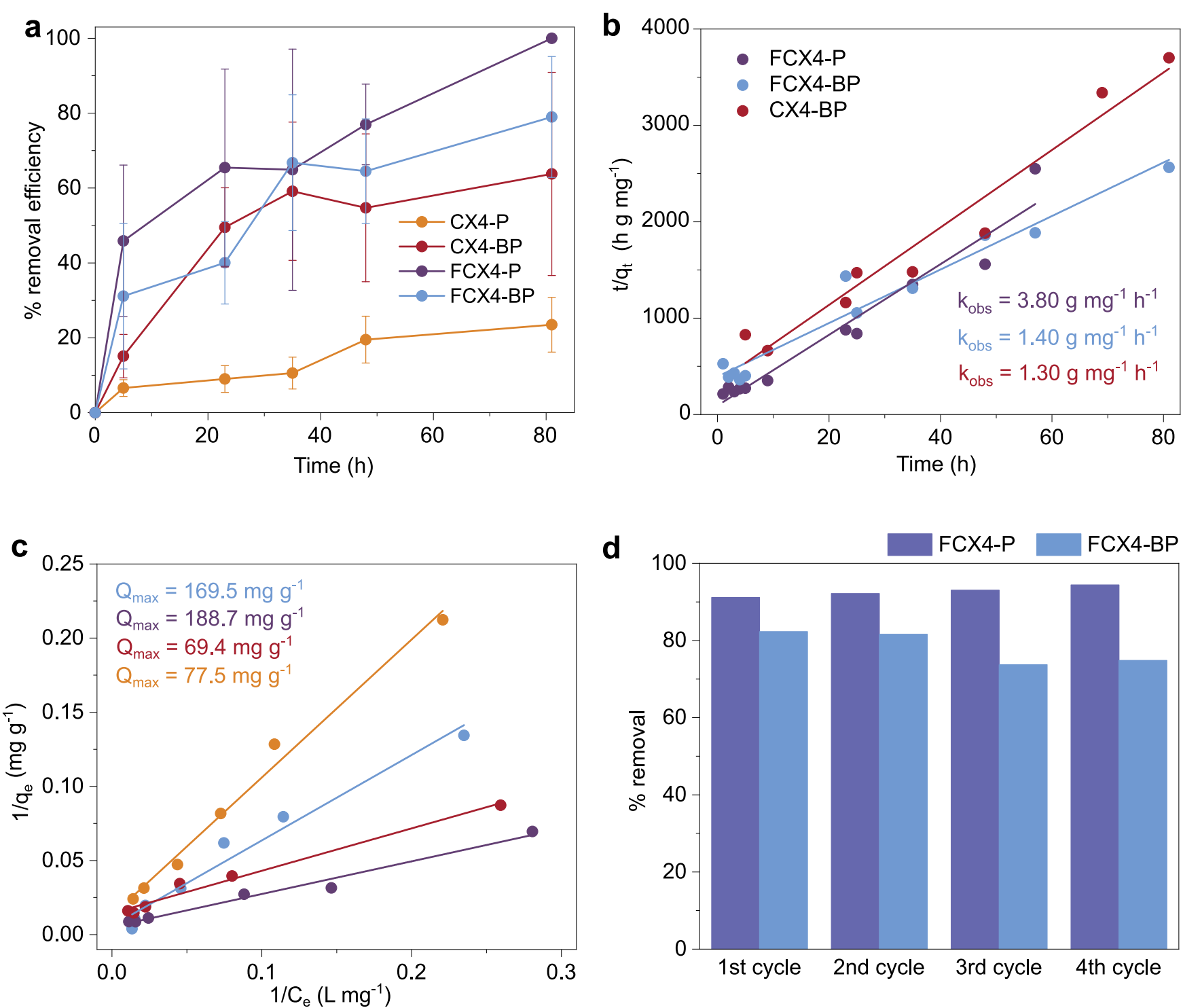

Figure 2. PFOA adsorption experiments. (a) Percent removal of PFOA over the course of 80 hours by single batches of FCX4-P, FCX4BP, CX4-P and CX4-BP. The error bars represent the average \% removal of three independent experiments. (b) Fitting of the kinetics data in panel (a) to pseudo-second order model of adsorption and the associated rate constants of adsorption ( $\mathrm{k}_{\mathrm{obs}}$ ); (c) linear forms of Langmuir isotherms along with calculated $Q_{\max }$ values for FCX4-P (purple), FCX4-BP (blue), CX4-P (red) and CX4-BP (orange); (d) regeneration experiments with FCX4-P and FCX4-BP demonstrate uncompromised PFOA removal efficiency in at least four adsorption cycles.

The PFOA adsorption experiments were conducted with vacuum-dried adsorbents at neutral $\mathrm{pH}$, and the concentration of PFOA was measured by HPLC-MS/MS (details in the SI). It was observed that fluorinated polymers $(79-100 \%$ removal $)$ performed much better than their non-fluorinated analogues (23 $-64 \%$ removal). Polymer FCX4-P was particularly efficient at removing PFOA as it reached close to $100 \%$ removal in 80 hours. We quantified the adsorption kinetics by using Ho and McKay's pseudo-second-order adsorption model, ${ }^{34}$ which enabled us to obtain the apparent rate constant $\left(\mathrm{k}_{\mathrm{obs}}\right)$ of $3.80 \mathrm{~g} \mathrm{mg}$ ${ }^{1} \mathrm{~h}^{-1}$ for FCX4-P, $1.40 \mathrm{~g} \mathrm{mg}^{-1} \mathrm{~h}^{-1}$ for FCX4-BP, $1.30 \mathrm{~g} \mathrm{mg}^{-1} \mathrm{~h}^{-}$ ${ }^{1}$ for CX4-BP, and $1.13 \mathrm{~g} \mathrm{mg}^{-1} \mathrm{~h}^{-1}$ for CX4-P (Figure 2b). It is difficult to compare these results to the majority of PFOA adsorbents reported in the literature, because they use initial PFOA concentrations that exceed environmentally relevant levels by several orders of magnitude (Table S1). Nevertheless, there are two reports using comparable concentrations of PFOA in kinetic experiments. A $\beta$-cyclodextrin polymer $^{19}$ and poly(ethylenimine)-functionalized cellulose microcrystals ${ }^{35}$ were reported to have $\mathrm{k}_{\text {obs }}$ values of $2.88 \mathrm{~g} \mathrm{mg}^{-1} \mathrm{~h}^{-1}$ and $0.213 \mathrm{~g}$ $\mathrm{mg}^{-1} \mathrm{~min}^{-1}$, respectively. However, these two materials suffer from relatively low maximum PFOA uptake capacities $\left(\mathrm{Q}_{\max }\right)$ of $33 \mathrm{mg} \mathrm{g}^{-1}$ and $2.32 \mathrm{mg} \mathrm{g}^{-1}$, respectively. We also investigated the probability of intra particle diffusion mechanism by plotting $\mathrm{Q}_{\mathrm{t}}$ as a function of $\mathrm{t}^{0.5}$ using the equation: $\mathrm{Q}_{\mathrm{t}}=\mathrm{K}_{\mathrm{p}} \mathrm{t}^{0.5}+\mathrm{C}$ where $\mathrm{K}_{\mathrm{p}}$ is the intra particle diffusion rate constant and $\mathrm{C}$ is the constant that define the boundary layer effect. ${ }^{36}$ For all the tested polymers we observed that there is a two-step adsorption process, an initial sharper one and a later more gradual one, which is controlled by particle diffusion (Figure S6).

In order to determine the maximum PFOA uptake capacity of our polymers, we conducted isotherm studies in which $5-100$ $\mathrm{mg} \mathrm{L}^{-1}$ PFOA solutions were incubated with the adsorbents until equilibrium was reached (details in Supporting Information). After quantifying the residual amounts of PFOA, the data was 
fitted to the Langmuir model of adsorption and $\mathrm{Q}_{\max }$ values were calculated (Figure 2c). FCX4-P, which was already found to have the fastest PFOA uptake in our polymer series, reached the highest $\mathrm{Q}_{\max }$ of $188.7 \mathrm{mg}$ PFOA adsorbed per gram of adsorbent. This value is almost six times higher than what is reported

\section{a}
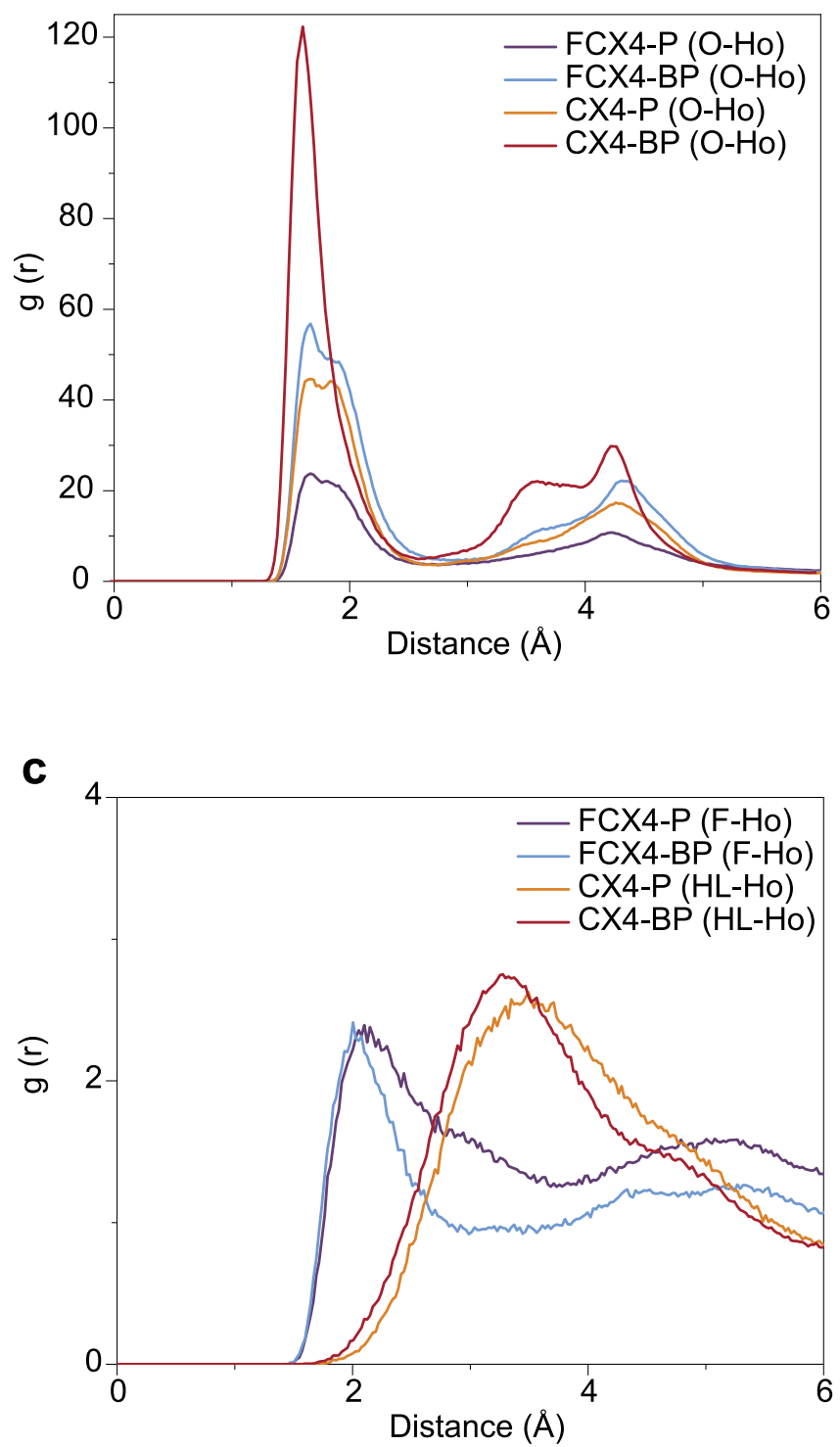

for the $\beta$-cyclodextrin-based polymer, ${ }^{19}$ nine times higher than the capacity of silica, ${ }^{37} 81$ times larger than $\mathrm{Q}_{\max }$ of poly(ethylenimine)-functionalized cellulose microcrystals ${ }^{35}$, and over

b
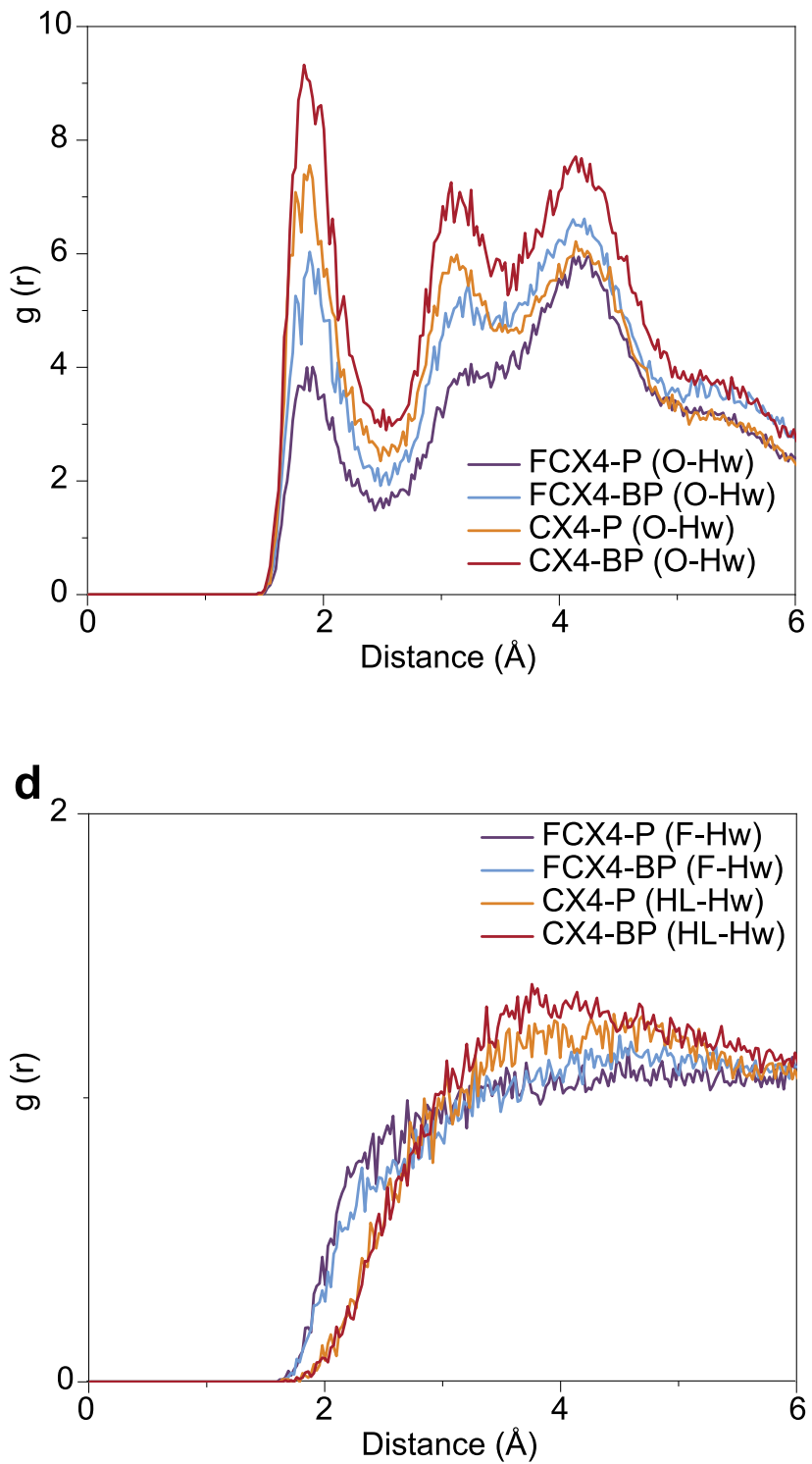

Figure 3. Radial distribution functions of specific interactions between the polymers and PFOA/water molecules. (a) O-Ho; (b) $\mathrm{O}-\mathrm{Hw}$; (c) HL-Ho and F-Ho; and (d) HL-Hw, F-Hw. (O is oxygen of calixarene in all polymers, HL is hydrogen of linkers in CX4-P and CX4-BP, F is fluorine of linkers in FCX4-P and FCX4-BP, Ho is carboxyl hydrogen of PFOA, and Hw is hydrogen of water. See Figure S7 for illustration of atom types and Figure S10 for simulation snapshots that illustrates PFOA-polymer interactions).

1,400 times higher than $\mathrm{Q}_{\max }$ of granulated activated carbon ${ }^{38}$ (Table S1). These results combined suggest that FCX4-P has one of the fastest PFOA adsorption kinetics at environmentally relevant concentrations and one of the highest maximum adsorption capacities for PFOA of any class of material. The performance of FCX4-BP is similar, with a $Q_{\max }$ of $169.5 \mathrm{mg} \mathrm{g}^{-1}$, while non-fluorinated polymers exhibited noticeably lower values of $69.4 \mathrm{mg} \mathrm{g}^{-1}$ and $77.5 \mathrm{mg} \mathrm{g}^{-1}$ for CX4-BP and CX4-P, respectively. The performance of this material, in comparison with known PFOA adsorbents, demonstrates the great potential of fluorinated calixarene polymers for this type of application.
A major advantage of the herein reported polymers for PFOA adsorption is their ability to be regenerated without compromising their adsorption capacity. Simple methanol washes for an extended period at room temperature were sufficient to regenerate FCX4-P and FCX4-BP (Figure 2d; details in Supporting information). Both materials retained their percent PFOA removal ability for the four consecutive tested cycles. FCX4-P removed 91, 92, 93, and $94 \%$ of the pollutant, while FCX4-BP removed $82,82,74$, and $75 \%$ of PFOA in four respective cycles. These results demonstrate the potential for long-term use 
of our porous fluorinated calixarene-based polymers for PFOA removal.

Given the findings of Omorodion et al., who studied the nature of non-covalent interactions in calixarene-PFOA hostguest complexes, ${ }^{29}$ we speculated that the primary interaction responsible for the successful adsorption of PFOA by the polymers is the hydrogen bond between the carboxylic group of PFOA and a hydroxyl oxygen of the calixarene. We also surmise that additional stabilization may be achieved by C-F $\cdots$ F$\mathrm{C}$ interactions ${ }^{39}$ between PFOA and the fluorinated linkers in FCX4-P and FCX4-BP. To test these hypotheses, we resorted to atomistic Monte Carlo (MC) simulations of PFOA and water. Polymer sheets representative of CX4-P, CX4-BP, FCX4-P, and FCX4-BP (see SI for details) were sampled for binding sites using a PFOA and a water molecule. Radial distribution functions (RDF) obtained from MC simulations allowed for comparisons to be made between the interactions of the polymers with PFOA/water (Figure 3). For all polymers, we found that hydrogen bonding is the main interaction between the polymer and its environment, and that the effect of C-F $\cdots \cdot \mathrm{F}-\mathrm{C}$ interaction is rather marginal (Figure S9).

The hydroxyl groups of the calixarenes are the preferential binding sites for both PFOA and water. For PFOA, this is evidenced by peaks near $1.8 \AA$ between the oxygen of the calixarenes and the carboxyl hydrogen of PFOA, denoted as OHo (Figure 3a). Similarly, water hydrogen bonds with the oxygen atoms of the calixarene ring, denoted as $\mathrm{O}-\mathrm{Hw}$ (Figure 3b). It is clear that both PFOA and water are expected to compete for the calixarene ring during adsorption for all polymers, whether fluorinated or not.

It was also found that fluorination leads to dramatic changes in the way the linkers interact with PFOA and water. In CX4-P and CX4-BP, the RDFs for the hydrogen atoms of the linkers and the carboxyl hydrogen atoms of the PFOA, denoted as HLHo, exhibited broad peaks around $3.4 \AA$ (Figure $3 \mathrm{c}$ ), which indicated that there were relatively weak interactions between PFOA and the linkers. For water, the interactions with the linkers are also limited, as such, the RDFs for the hydrogen atoms of the linkers and the hydrogen atoms of water, denoted as HLHw, showed only weak structuring around $3.8 \AA$ (Figure $3 \mathrm{~d}$ ). In FCX4-P and FCX4-BP, however, substitution of the linker hydrogen atoms with fluorine atoms resulted in tighter PFOA binding to the linkers. This is demonstrated by the peaks observed at $2 \AA$ in the RDFs for the fluorine atoms of the linkers and the carboxyl hydrogen atoms of the PFOA, denoted as FHo (Figure $3 \mathrm{c}$ ). To understand the nature of this affinity, we computed the binding energy of PFOA to the polymers (see SI for details). Indeed, energy calculations revealed that fluorination increases the binding strength of PFOA to the linkers (see SI for details). The binding energy was reduced by 3.8 and 2.4 $\mathrm{kJ} / \mathrm{mol}$ for FCX4-P and FCX4-BP, respectively, in accordance with the experimental observations and RDF analysis. On the other hand, after fluorination, water-linker interactions became weaker, as the RDFs between the fluorine atoms of the linkers and the hydrogen atoms of water, denoted as F-Hw, did not show any structuring (Figure $3 \mathrm{~d}$ ). This also suggests that fluorine rich PFOA molecules, when adsorbed, can exclude water adsorption in the pores. All in all, our simulations provide strong evidence that fluorination creates new sites on the linkers for the adsorption of PFOA, which no longer needs to compete with water. In addition, fluorination stabilizes PFOA at the new binding site; resulting in enhanced PFOA binding to FCX4-P and FCX4-BP, as compared to the non-fluorinated polymers, CX4-P and CX4-BP.

\section{CONCLUSIONS}

In summary, we have developed two fluorine-rich calix[4]arene-based porous polymers and evaluated their efficiency for removing PFOA from water. Polymer FCX4-P removed PFOA more efficiently than any other adsorbent tested and exhibited a very high uptake capacity along with fast adsorption kinetics. By comparing its performance with nonfluorinated calixarene polymers, we conclude that the development of favorable C-F $\cdots$ F-C interactions between PFOA and the fluorinated linkers are essential for the excellent performance of our material. Additionally, PFOA adsorption was found to be reversible, as PFOA can be desorbed by simple methanol washing, and the polymers were able to be recycled without significant performance loss. Monte Carlo simulations used to understand the mechanism of PFOA adsorption, highlight the importance of hydrogen bonding between the carboxylic group of PFOA and the hydroxyl groups of calixarenes, and the role of hydrophobic interactions between fluorinated linkers and PFOA. The latter explained the higher affinity of fluorinated polymers for PFOA. Efforts are currently underway to explore the potential large-scale applicability of fluorinated calixarene polymers towards target-specific molecular sequestration and environmental remediation of waters. Our findings illustrate that macrocycle-based porous polymers are a viable alternative technology for water decontamination with horizons trending towards applications for the purification of valuable multi-sourced products.

\section{ASSOCIATED CONTENT}

\section{Supporting Information}

The Supporting Information is available free of charge on the ACS Publications website.

\section{AUTHOR INFORMATION}

\section{Corresponding Author}

* Ali Trabolsi, ali.trabolsi@nyu.edu

\section{Present Addresses}

\# Current address: Department of Chemistry, University of Cambridge, Lensfield Road, Cambridge CB2 1EW, UK.

\section{Author Contributions}

$\dagger$ These authors contributed equally.

Notes

The authors declare no competing interests.

\section{ACKNOWLEDGMENTS}

The research described here was sponsored by New York University Abu Dhabi (NYUAD), UAE. The authors thank NYUAD for its generous support of the research program at NYUAD. DS acknowledges the financial support from the Khalifa Universityfaculty startup grant (FSU-2020) and the support from Khalifa University under the RCII-2018-024.. SK would like to thank for the AD181 faculty research grant. The research was carried out by using the Core Technology Platform resources at NYUAD. The au- 
thors acknowledge the use of the University College London, Myriad High Throughput Computing Facility (Myriad@UCL), and associated support services, in the completion of this work.

\section{REFERENCES}

(1) Trager, R. A Persistent Perfluorinated Problem. Chem. World 2019, No. August, 26-31.

(2) Gorrochategui, E.; Pérez-Albaladejo, E.; Casas, J.; Lacorte, S.; Porte, C. Perfluorinated Chemicals: Differential Toxicity, Inhibition of Aromatase Activity and Alteration of Cellular Lipids in Human Placental Cells. Toxicol. Appl. Pharmacol. 2014, 277 (2), 124-130.

Ahrens, L.; Norström, K.; Viktor, T.; Cousins, A. P.; Josefsson, S. Stockholm Arlanda Airport as a Source of Per-and Polyfluoroalkyl Substances to Water, Sediment and Fish. Chemosphere 2015, 129, 33-38.

(4) Lau, C.; Anitole, K.; Hodes, C.; Lai, D.; Pfahles-Hutchens, A.; Seed, J. Perfluoroalkyl Acids: A Review of Monitoring and Toxicological Findings. Toxicol. Sci. 2007, 99 (2), 366-394.

(5) Hori, H.; Nagano, Y.; Murayama, M.; Koike, K.; Kutsuna, S. Efficient Decomposition of Perfluoroether Carboxylic Acids in Water with a Combination of Persulfate Oxidant and Ultrasonic Irradiation. J. Fluor. Chem. 2012, 141, 5-10.

(6) Huang, L.; Dong, W.; Hou, H. Investigation of the Reactivity of Hydrated Electron toward Perfluorinated Carboxylates by Laser Flash Photolysis. Chem. Phys. Lett. 2007, 436 (1-3), 124-128.

(7) Thi, L.-A. P.; Do, H.-T.; Lee, Y.-C.; Lo, S.-L. Photochemical Decomposition of Perfluorooctanoic Acids in Aqueous Carbonate Solution with UV Irradiation. Chem. Eng. J. 2013, 221, 258-263.

(8) Carter, K. E.; Farrell, J. Oxidative Destruction of Perfluorooctane Sulfonate Using Boron-Doped Diamond Film Electrodes. Environ. Sci. Technol. 2008, 42 (16), 6111-6115.

(9) Javed, H.; Lyu, C.; Sun, R.; Zhang, D.; Alvarez, P. J. J. Discerning the Inefficacy of Hydroxyl Radicals during Perfluorooctanoic Acid Degradation. Chemosphere 2020, 247 (125883), 1-6.

(10) Du, Z.; Deng, S.; Bei, Y.; Huang, Q.; Wang, B.; Huang, J.; Yu, G. Adsorption Behavior and Mechanism of Perfluorinated Compounds on Various Adsorbents-a Review. J. Hazard. Mater. 2014, 274, 443-454.

(11) Jantzen, C. E.; Toor, F.; Annunziato, K. A.; Cooper, K. R. Effects of Chronic Perfluorooctanoic Acid (PFOA) at Low Concentration on Morphometrics, Gene Expression, and Fecundity in Zebrafish (Danio Rerio). Reprod. Toxicol. 2017, 69, 34-42.

(12) Ahrens, L. Polyfluoroalkyl Compounds in the Aquatic Environment: A Review of Their Occurrence and Fate. $J$. Environ. Monit. 2011, 13 (1), 20-31.

(13) Ateia, M.; Alsbaiee, A.; Karanfil, T.; Dichtel, W. R. Efficient PFAS Removal by Amine-Functionalized Sorbents: Critical Review of the Current Literature. Environ. Sci. Technol. Lett. 2019, 6 (12), 688-695.

(14) Ochoa-Herrera, V.; Sierra-Alvarez, R. Removal of Perfluorinated Surfactants by Sorption onto Granular Activated Carbon, Zeolite and Sludge. Chemosphere 2008, 72 (10), 1588-1593.

(15) Yu, Q.; Zhang, R.; Deng, S.; Huang, J.; Yu, G. Sorption of Perfluorooctane Sulfonate and Perfluorooctanoate on Activated Carbons and Resin: Kinetic and Isotherm Study. Water Res. 2009, 43 (4), 1150-1158.

(16) Kumarasamy, E.; Manning, I. M.; Collins, L. B.; Coronell, O.; Leibfarth, F. A. Ionic Fluorogels for Remediation of Per- and Polyfluorinated Alkyl Substances from Water. ACS Cent. Sci. 2020, 6 (4), 487-492. https://doi.org/10.1021/acscentsci.9b01224.

(17) Lu, W.; Yuan, D.; Zhao, D.; Schilling, C. I.; Plietzsch, O.; Muller, T.; Bräse, S.; Guenther, J.; Blümel, J.; Krishna, R. Porous Polymer Networks: Synthesis, Porosity, and Applications in Gas Storage/Separation. Chem. Mater. 2010, 22 (21), 5964-5972.

(18) Talapaneni, S. N.; Kim, D.; Barin, G.; Buyukcakir, O.; Je, S. H.; Coskun, A. Pillar[5]Arene Based Conjugated Microporous Polymers for Propane/Methane Separation through Host-Guest Complexation. Chem. Mater. 2016, 28 (12), 4460-4466.

(19) Xiao, L.; Ling, Y.; Alsbaiee, A.; Li, C.; Helbling, D. E.; Dichtel, W. R. $\beta$-Cyclodextrin Polymer Network Sequesters
Perfluorooctanoic Acid at Environmentally Relevant Concentrations. J. Am. Chem. Soc. 2017, 139 (23), 7689-7692. Xiao, L.; Ching, C.; Ling, Y.; Nasiri, M.; Klemes, M. J.; Reineke, T. M.; Helbling, D. E.; Dichtel, W. R. Cross-Linker Chemistry Determines the Uptake Potential of Perfluorinated Alkyl Substances by $\beta$-Cyclodextrin Polymers. Macromolecules 2019 , 52 (10), 3747-3752.

(21) Li, C.; Klemes, M. J.; Dichtel, W. R.; Helbling, D. E. Tetrafluoroterephthalonitrile-Crosslinked Beta-Cyclodextrin Polymers for Efficient Extraction and Recovery of Organic Micropollutants from Water. J. Chromatogr. A 2018, 1541 (March), 52-56.

(22) Alzate-Sanchez, D. M.; Ling, Y.; Li, C.; Frank, B. P.; Bleher, R.; Fairbrother, D. H.; Helbling, D. E.; Dichtel, W. R. $\beta$-Cyclodextrin Polymers on Microcrystalline Cellulose as a Granular Media for Organic Micropollutant Removal from Water. ACS Appl. Mater. Interfaces 2019, 11 (8), 8089-8096.

(23) Byun, J.; Patel, H. A.; Thirion, D.; Yavuz, C. T. Charge-Specific Size-Dependent Separation of Water-Soluble Organic Molecules by Fluorinated Nanoporous Networks. Nat. Commun. 2016, 7 (1), $1-10$.

(24) Kim, S.; Thirion, D.; Nguyen, T. S.; Kim, B.; Dogan, N. A.; Yavuz, C. T. Sustainable Synthesis of Superhydrophobic Perfluorinated Nanoporous Networks for Small Molecule Separation. Chem. Mater. 2019, 31 (14), 5206-5213.

(25) Shin, H.; Kim, D.; Kim, H. J.; Kim, J.; Char, K.; Yavuz, C. T. Choi, J. W. Fluorinated Covalent Organic Polymers for High Performance Sulfur Cathodes in Lithium-Sulfur Batteries. Chem. Mater. 2019, 31 (19), 7910-7921.

(26) Patel, H. A.; Je, S. H.; Park, J.; Chen, D. P.; Jung, Y.; Yavuz, C. T.; Coskun, A. Unprecedented High-Temperature CO2 Selectivity in N2-Phobic Nanoporous Covalent Organic Polymers. Nat. Commun. 2013, 4 (1), 1-8.

(27) Liu, G.; Wang, Y.; Shen, C.; Ju, Z.; Yuan, D. A Facile Synthesis of Microporous Organic Polymers for Efficient Gas Storage and Separation. J. Mater. Chem. A 2015, 3 (6), 3051-3058.

(28) Palkovits, R.; Antonietti, M.; Kuhn, P.; Thomas, A.; Schüth, F. Solid Catalysts for the Selective Low-temperature Oxidation of Methane to Methanol. Angew. Chemie Int. Ed. 2009, 48 (37), 6909-6912.

(29) Omorodion, H.; Palenzuela, M.; Ruether, M.; Twamley, B.; Platts, J. A.; Baker, R. J. A Rationally Designed Perfluorinated Host for the Extraction of PFOA from Water Utilising NonCovalent Interactions. New J. Chem. 2018, 42 (10), 7956-7968.

(30) Shetty, D.; Skorjanc, T.; Raya, J.; Sharma, S. K.; Jahovic, I. Polychronopoulou, K.; Asfari, Z.; Han, D. S.; Dewage, S.; Olsen, J.-C.; Jagannathan, R.; Kirmizialtin, S.; Trabolsi, A. Calix[4]Arene-Based Porous Organic Nanosheets. ACS Appl. Mater. Interfaces 2018, 10 (20), 17359-17365.

(31) Shetty, D.; Jahovic, I.; Raya, J.; Asfari, Z.; Olsen, J.-C.; Trabolsi, A. Porous Polycalix[4]Arenes for Fast and Efficient Removal of Organic Micropollutants from Water. ACS Appl. Mater. Interfaces 2018, 10 (3), 2976-2981.

(32) Neenan, T. X.; Whitesides, G. M. Synthesis of High Carbon Materials from Acetylenic Precursors. Preparation of Aromatic Monomers Bearing Multiple Ethynyl Groups. J. Org. Chem. 1988, 53 (11), 2489-2496.

(33) World Health Organization. Keeping Our Water Clean: The Case of Water Contamination in the Veneto Region, Italy; 2017.

(34) Ho, Y.-S.; McKay, G. Pseudo-Second Order Model for Sorption Processes. Process Biochem. 1999, 34 (5), 451-465.

(35) Ateia, M.; Attia, M. F.; Maroli, A.; Tharayil, N.; Alexis, F.; Whitehead, D. C.; Karanfil, T. Rapid Removal of Poly- and Perfluorinated Alkyl Substances by Poly(Ethylenimine)Functionalized Cellulose Microcrystals at Environmentally Relevant Conditions. Environ. Sci. Technol. Lett. 2018, 5 (12), 764-769.

(36) Fierro, V.; Torne'-Ferna'ndez, V.; Montane', D.; Celzard, A. Adsorption of phenol onto activated carbons having different textural and surface properties. Microporous and Mesoporous Mater. 2008, 276-284.

(37) Stebel, E. K.; Pike, K. A.; Nguyen, H.; Hartmann, H. A.; Klonowski, M. J.; Lawrence, M. G.; Collins, R. M.; Hefner, C. E.; Edmiston, P. L. Absorption of Short-Chain to Long-Chain Perfluoroalkyl Substances Using Swellable Organically Modified 
Silica. Environ. Sci. Water Res. Technol. 2019, 5, 1854-1866.

(38) Zhang, D.; Luo, Q.; Gao, B.; Chiang, S.-Y. D.; Woodward, D.; Huang, Q. Sorption of Perfluorooctanoic Acid, Perfluorooctane Sulfonate and Perfluoroheptanoic Acid on Granular Activated Carbon. Chemosphere 2016, 144, 2336-2342.

(39) Baker, R. J.; Colavita, P. E.; Murphy, D. M.; Platts, J. A.; Wallis, J. D. Fluorine-Fluorine Interactions in the Solid State: An
Experimental and Theoretical Study. J. Phys. Chem. A 2011, 116 (5), 1435-1444. 
Table of Contents

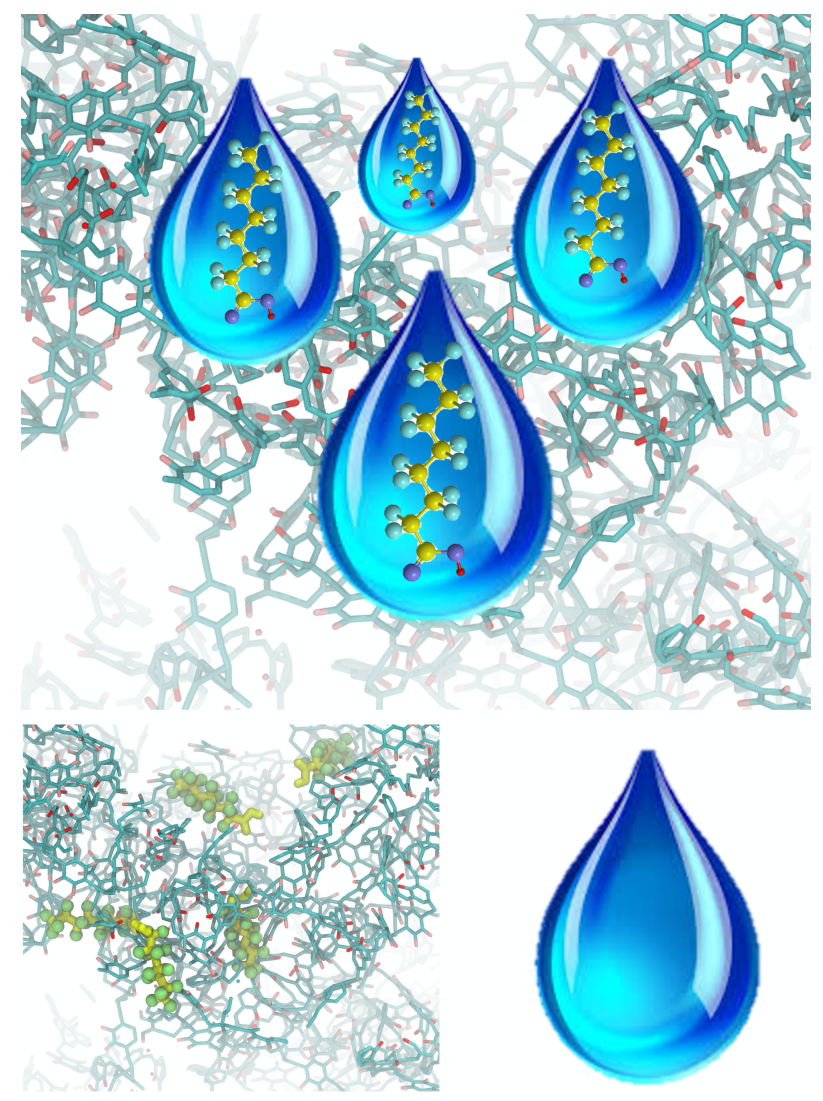

